\title{
Effective segmentation method of target based on polarization- modulated 3D imaging system (Erratum)
}

Shengjie Wang, Bo Liu, Heping Li, Shenglin Lv

Shengjie Wang, Bo Liu, Heping Li, Shenglin Lv, "Effective segmentation method of target based on polarization-modulated 3D imaging system (Erratum)," Proc. SPIE 11434, 2019 International Conference on Optical Instruments and Technology: Optical Systems and Modern Optoelectronic Instruments, 114342M (10 April 2020); doi: 10.1117/12.2572264

SPIE Event: 2019 International Conference on Optical Instruments and Technology, 2019, Beijing, China 


\section{Effec tive segmentation method of target based on polarization-modulated 3D imaging system (Eratum)}

Shengjie Wang1,2,3, Bo Liu1,3, Heping Lí2 and Shenglin Lv4

${ }^{1}$ Key Lab of Space Optoelectronic Precision Measurement Technology (China)

2 University of Electronic Science and Technology of China (China)

3 Institute of Optics and Electronic S (China)

${ }^{4}$ Chinese AirForce (China)

Proceedings Volume 11434, 2019 Intemational Conference on Optic al Instruments and Technology: Optical Systems and Modem Optoelectronic Instruments; 114341M (2020) https:// doi.org/10.1117/12.2557409

Event 2019 Intemational Conference on Optical Instruments and Technology (OIT2019), 2019, Beijing, China

Online Publication Date: 12 March 2020

Enatum Published: 13 Ap ril 2020

A revised version of this manuscript was published on 13 April 2020. Details of the revision are provided in the text that accompanies this Erratum. The original paper has been updated. 